\title{
Lid-driven cavity flow using dual reciprocity
}

\author{
Juraj Mužík ${ }^{1, *}$ and Roman Bulko ${ }^{1}$ \\ ${ }^{1}$ University of Žilina, Department of geotechnics, Univerzitná 8215/1, 01026 Žilina, Slovak Republic
}

\begin{abstract}
The paper presents the use of the multi-domain dual reciprocity method of fundamental solutions (MD-MFSDR) for the analysis of the laminar viscous flow problem described by Navier-Stokes equations. A homogeneous part of the solution is solved using the method of fundamental solutions with the 2D Stokes fundamental solution - Stokeslet. The dual reciprocity approach has been chosen because it is ideal for the treatment of the non-homogeneous and nonlinear terms of Navier-Stokes equations. The presented DR-MFS approach to the solution of the 2D flow problem is demonstrated on a standard benchmark problem - lid-driven cavity.
\end{abstract}

\section{Introduction}

The solution of Navier-Stokes (NS) equations is one of the basic tasks of computational fluid mechanics. This nonlinear system of differential equations has already been solved by several numerical methods, starting with the finite difference method through the finite element method to meshless and boundary type methods. The methods based on boundary integral theory are represented by the local boundary integral element method (LBIEM) [1], the boundary element method (BEM) $[2,3]$. The method of fundamental solutions (MFS) presented in this article belongs to the class of so-called Trefftz methods.

In the case of BEM the singularities of the fundamental solution are handled by proper integration method, the MFS overcomes the singularity using a fictitious boundary, but the optimum location of this boundary remains the open problem especially for complexshaped domains.

To handle the non-linear and non-homogeneous part of the NS equation solution, which is not captured by the fundamental solution, the Dual Reciprocity Method (DRM) approach is adopted. The DRM approximates the non-linear and non-homogeneous terms in the particular part of the solution as a series of known interpolation functions that depend only upon the geometry of the problem. In the proposed formulation the DRM used a regular set of collocation nodes distributed inside the sub-domain interior and along the boundary to approximate the non-linear terms.

In the present work, the DRM global nature has been altered by partitioning the computational domain into smaller sub-domains or domain elements and on each of them, the dual reciprocity method was applied to obtain an expression for nonlinear terms. The

* Corresponding author: juraj.muzik@uniza.sk 
continuity of the solution over the boundary of adjacent sub-domains is enforced applying the equality of velocities and tractions on the mutual boundary.

The numerical example using the multi-domain technique for the traditional benchmark problem of lid-driven cavity flow will be shown. The agreement between the results of the proposed technique and the results of other authors is excellent.

\section{Method of fundamental solutions - MFS}

The MFS implementation is very straightforward and only a little boundary data preparation is required in terms of boundary collocation or boundary fitting which is simpler than the domain-type methods (FEM, FDM). Although the MFS has been successfully applied to a variety of complicated problems [4] there have been no applications of this method investigation of laminar flow problems.

The method of fundamental solutions is equivalent to modified indirect Trefftz formulation. In this formulation, the approximate solution is represented by the linear combination of the singular fundamental solution and then, the unknown scaling parameters are determined so that the approximate solution satisfies the boundary conditions by the means of the collocation method. This formulation was named as a method of fundamental solutions.

Before the full formulation for the solution of Navier-Stokes (NS) equations, we start with a similar problem that can be described by the linear partial differential equation for which the fundamental solution exists - Stokes flow. Stokes flow is governed by the equation similar to NS but not accounting for nonlinear convection effect, which becomes significant for high Reynolds number flows. The Stokes flow PDE is formulated as follows

$$
\mu \frac{\partial^{2} u_{i}}{\partial x_{j} \partial x_{j}}-\frac{\partial p}{\partial x_{i}}=f_{i} ; \quad \frac{\partial u_{i}}{\partial x_{i}}=0
$$

where $u, p, \mu$ is velocity, pressure, and dynamic viscosity respectively. The corresponding fundamental solution of the two-dimensional Stokes problem is given as

$$
G_{i j}(P, Q)=-\frac{1}{4 \pi}\left(\ln \left(\frac{1}{|P-Q|}\right) \delta_{i j}+\frac{\left(P_{i}-Q_{i}\right)\left(P_{j}-Q_{j}\right)}{|P-Q|^{2}}\right)
$$

where $P$ and $Q$ are the response and the source points, respectively and $|P-Q|$ is the Euclidean distance between the points $P$ and $Q$. The fundamental solution has the singularity at $P=Q$. Thus, the source points are placed on the imaginary boundary surrounding the (real) boundary outside the solution domain. The solution is then approximated as follows

$$
u_{i j}(P) \cong \tilde{u}_{i j}(P)=\sum_{k=1}^{n} \alpha_{i j}^{k} G_{i j}\left(P, Q^{k}\right)
$$

where $n$ is the total number of the source points. Since equation (3) do not satisfy the boundary conditions on the real boundary $\Gamma$, the residual yield

$$
P \in \Gamma: R_{i j}=\tilde{u}_{i j}-\bar{u}_{i j}=\sum_{k=1}^{n} \alpha_{i j}^{k} G_{i j}\left(P, Q^{k}\right)-\bar{u}_{i j}
$$

In the collocation-method formulation (MFS), equation (4) is forced to vanish at the boundary collocation points. So we have following linear system of equations 


$$
\mathbf{A} \alpha=\mathbf{b}
$$

This linear system of equation is solved for unknown coefficients $\boldsymbol{\alpha}$. Using equation (3) can be then used to evaluate unknown velocity tensor at any point in the solution domain.

The singularity of the fundamental solution in the MFS is isolated by moving the sources outside the solution domain. The imaginary boundary used for sources doesn't need to have the same shape as the solution domain (see Fig. 1).

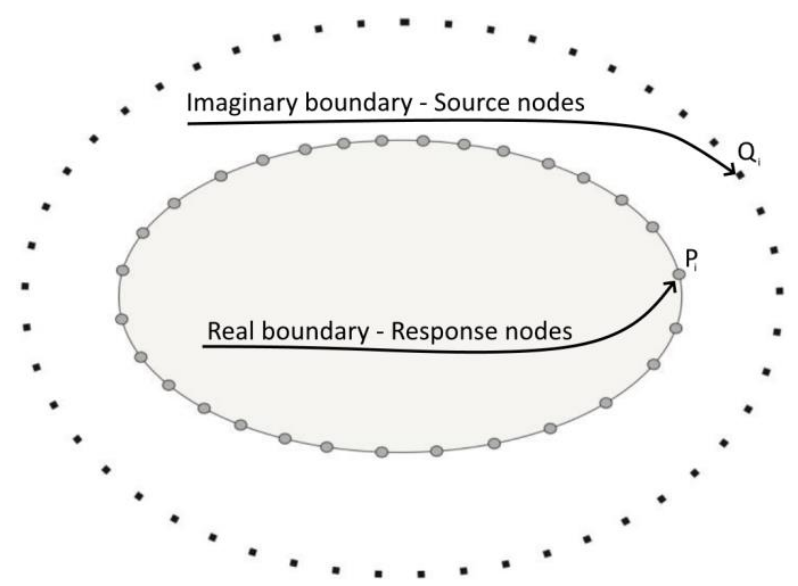

Fig. 1. Concept of the imaginary boundary used in MFS to isolate the singularity.

The problematic nature of the imaginary boundary in the MFS is problematic because its position affects the results and there is no rigorous way how to choose the position for this boundary. Various empirical approaches have been developed [4], but none of them have general applicability. Some new techniques have recently been developed to cure this problem of MFS, such as the boundary knot method, singular boundary method, and regularized meshless method.

\section{Dual reciprocity method}

To solve the flow governed by full NS equations there is a need for a technique that is capable to solve the non-linear and non-homogeneous terms. The NS equation can be described as follows

$$
\frac{\partial u_{i}}{\partial x_{i}}=0 ; \mu \frac{\partial^{2} u_{i}}{\partial x_{j} \partial x_{j}}-\frac{\partial p}{\partial x_{i}}=\rho \frac{\partial}{\partial x_{j}}\left(u_{j} u_{i}\right)+f_{i}
$$

where, $u, p, \mu$, and $\rho$ are velocity, pressure, dynamic viscosity, and density respectively. The solution of (6) in the context of dual reciprocity and method of fundamental solutions, can be defined as the sum of homogeneous and particular solutions

$$
u_{i j}=u_{i j}^{\mathrm{H}}+u_{i j}^{\mathrm{P}}, p_{i}=p_{i}^{H}+p_{i}^{P}
$$

where $u_{i j}{ }^{H}$ and $p_{i}{ }^{\mathrm{H}}$ is the velocity and pressure solutions of the homogeneous problem that captures the Stokes equations (1) together with the prescribed boundary conditions, and $u_{i j}{ }^{\mathrm{P}}$ and $p_{i}{ }^{\mathrm{P}}$ are the particular solutions of the non-homogeneous and non-linear equations (6). The homogeneous problem can be solved using the method of fundamental solutions (MFS) as was described in chapter 2, and the particular solution is constructed using the 
method of approximated particular solutions (MAPS). The particular solutions $u_{i j}{ }^{\mathrm{P}}$, and $p_{i}{ }^{\mathrm{P}}$ of the (6) is then constructed using a linear combination of the radial basis functions [2]

$$
u_{i j}^{\mathrm{P}}=\sum_{k=1}^{m} \beta_{i j}^{k} \bar{u}_{i j}\left(x^{k}\right), p_{i}^{P}=\sum_{k=1}^{m} \gamma_{i}^{k} \bar{p}_{i}\left(x^{k}\right)
$$

where $m$ is the number of the internal nodes in the sub-domain (see Fig. 2), $\beta_{\mathrm{ij}}$ and $\gamma_{\mathrm{i}}$ are the unknown coefficients, and $\bar{u}$ and $\bar{p}$ are the globally defined radial basis functions that are used to construct the particular solutions corresponding to the following non-homogeneous and non-linear system of equations $[2,5]$

$$
\mu \frac{\partial^{2} \bar{u}_{i k}}{\partial x_{j} \partial x_{j}}-\frac{\partial \bar{p}_{k}}{\partial x_{i}}=\varphi(x) \delta_{i k} ; \frac{\partial \bar{u}_{i k}}{\partial x_{i}}=0
$$

where $\varphi(x)$ is the globally defined unity radial basis function, with the assumption of the centre at the beginning of coordinate system $x=(0,0)$

$$
\varphi(x)=1
$$

One of the main difficulties is how to find a closed-form expression of the particular solutions $\bar{u}_{i k}$ and $\bar{p}_{k}$. To solve the problem, we employ the way more detailed described in [5]. As the first step, yet unknown auxiliary potential $\Phi$ is defined and is used to express the velocity particular solution as follows

$$
\bar{u}_{i k}=\frac{\partial^{2} \Phi}{\partial x_{j} \partial x_{j}} \delta_{i k}-\frac{\partial^{2} \Phi}{\partial x_{i} \partial x_{k}}
$$

The velocity particular solution defined using equation (11) satisfies implicitly the continuity equation. Substituting (11) into the momentum equation (9) allows one to write

$$
\mu\left(\frac{\partial^{4} \Phi}{\partial x_{j} \partial x_{j} \partial x_{k} \partial x_{k}} \delta_{i k}-\frac{\partial^{4} \Phi}{\partial x_{j} \partial x_{j} \partial x_{i} \partial x_{k}}\right)-\frac{\partial \bar{p}_{k}}{\partial x_{i}}=\varphi \delta_{i k}
$$

Splitting the equation (12) into two parts

$$
\begin{gathered}
\mu \frac{\partial^{4} \Phi}{\partial x_{j} \partial x_{j} \partial x_{k} \partial x_{k}}=\varphi(x) \\
\mu \frac{\partial^{4} \Phi}{\partial x_{j} \partial x_{j} \partial x_{i} \partial x_{k}}+\frac{\partial \bar{p}_{k}}{\partial x_{i}}=0
\end{gathered}
$$

allows one to evaluate the unknown auxiliary potential from equation (13) by direct integration, having the radial dependence of function (10) in mind.

$$
\Phi(x)=\frac{r^{4}}{64} ; \quad r=|x|
$$

The second equation, (14) is used to obtain pressure particular solution

$$
\bar{p}_{k}=\mu \frac{\partial^{3} \Phi}{\partial x_{j} \partial x_{j} \partial x_{k}}
$$

Substituting the auxiliary potential $\Phi$ into the equations (16) and (11) the pressure and velocity field of a particular solution is obtained. 


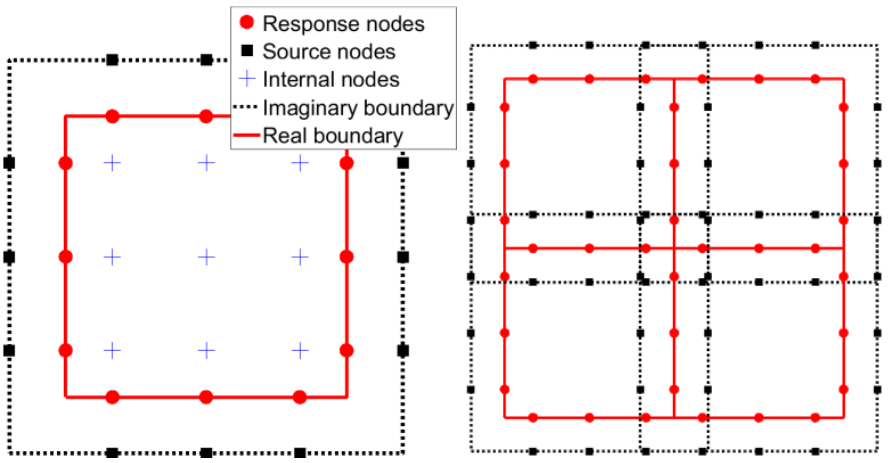

Fig. 2. Detail of the single sub-domain and computational sub-domain mesh.

\section{Simulation of the lid-driven cavity problem}

Lid-driven cavity flow is being used as a standard test case for the validation of numerical techniques of laminar incompressible Navier-Stokes flow. The lid of the cavity moves at a constant velocity $u=1$, and no-slip boundary conditions are prescribed for the remaining walls of the square computational domain. The geometrical configuration and prescribed boundary conditions are shown in Fig. 3. The challenge of this benchmark problem, in the context of the numerical method, is the singularity of the pressure and velocity solution at the top corners of the cavity. Therefore, the size of the computational sub-domains utilized decreases towards the corners of the cavity (see Fig. 3) to catch the high gradients of pressure and velocity solutions.

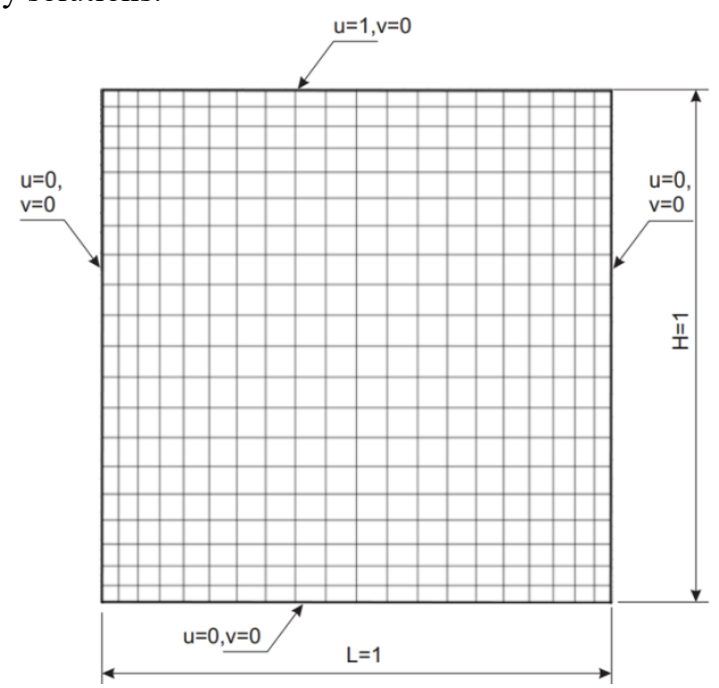

Fig. 3. The lid-driven cavity problem geometry, boundary conditions, and distribution of internal cells (sub-domains). 

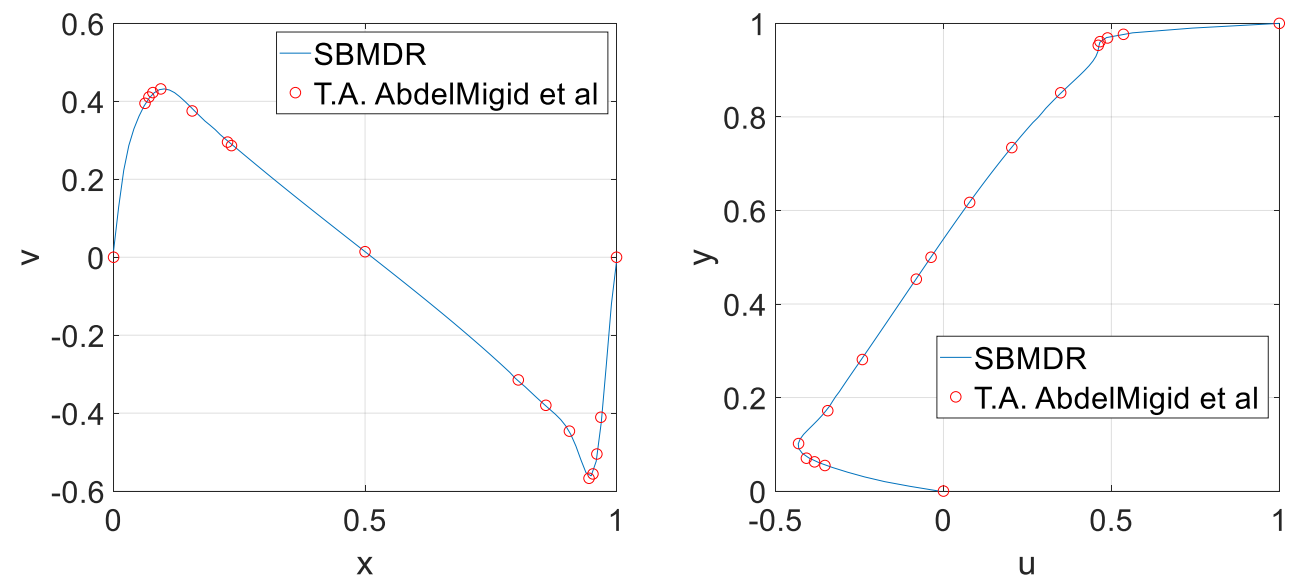

Fig. 4. Lid-driven cavity, $\operatorname{Re}=3200$, left: vertical velocity components along horizontal line $y=0.5$, right: horizontal velocity components along vertical line $x=0.5$.
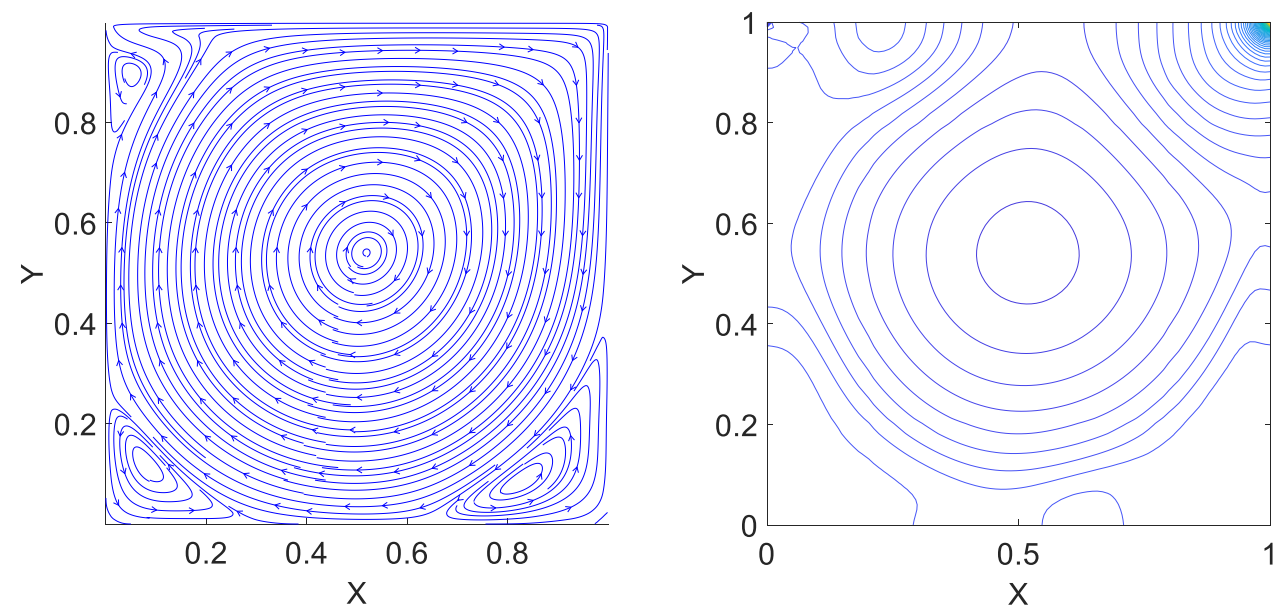

Fig. 5. Streamlines and Pressure contours for Lid-driven cavity problem, $\operatorname{Re}=3200$.

The results of the MD-MFSDR are confronted with the solution represented in [6] for the Reynolds number $\mathrm{Re}=3200$; the vertical velocity components along a horizontal line and the horizontal velocity components along a vertical line, straight to the centre of the cavity, are plotted in Fig. 4. Fig. 5 shows streamlines and pressure contours for Reynolds number of 3200 .

\section{Conclusions}

This paper presents the solution of the incompressible laminar flow governed by a primitive variable formulation of the Navier-Stokes equation using the method of fundamental solution. Lid driven cavity benchmarking problem has been successfully tested. This type of boundary collocation method seems to be a quite useful alternative to the solutions of incompressible fluid flow using the boundary-based numerical schemes. The dual reciprocity technique implemented in conjunction with the sub-domain approach generates a sparse characteristic matrix and enables the method to obtain reasonable results even for 
high Reynolds numbers. The presented numerical code and numerical results need to be more analyzed in the sense of precision, convergence and computational demands.

This contribution is the result of the project funded by the Scientific Grant Agency of Slovak Republic (VEGA) No. 1-0716-17.

\section{References}

1. K. Kovářík, D. Sitányiová, J. Mužík, Eng. Anal. Bound. Elem. 44, 55 (2014)

2. C.A. Brebbia, Boundary Element Techniques in Engineering (Elsevier, 2016)

3. P. Partridge, Dual Reciprocity Boundary Element Method (Springer Science \& Business Media, 2012)

4. J. Mužík, R. Bulko, Free surface groundwater flow solution using boundary collocation methods, in XXVII R-S-P Seminar, TFoCE, 17-21 September 2018, Rostov-on-Don, Russia (2018)

5. W.F. Florez, Nonlinear flow using dual reciprocity (WIT Press, 2001)

6. T.A. AbdelMigid, K.M. Saqr, M.A. Kotb, A.A. Aboelfarag, Alex. Eng. J. 56, 123 (2016) 